\title{
NEURAL NETWORK APPROACH TO THE ENHANCEMENT OF EEG SIGNALS IN THE PRESENCE OF EOG ARTEFACTS
}

\author{
P. K. Sadasivan and D. Narayana Dutt \\ Department of Electrical Communication Engg. \\ Indian Institute of Science \\ Bangalore-560 012. INDIA
}

\begin{abstract}
Human electroencephalogram (EEG) contains useful diagnostic information on a variety of neurological disorders. However, like all biomedical signals, EEG is also contaminated with many 1, unwanted signals or artefacts which seriously affects its clinical usefulness. One of the'main disturbances is due to the eye movements, which generate an electrical activity called electrooculogram (EOG). In this paper, we propose a Neural Network (NN) approach to the enhancement of EEG signals in the presence of EOG artefacts. We recast the EEG enhancement problem into the optimization framework by developing an appropriate cost function. The cost function is nothing but the energy in the enhanced EEG signal which is obtained through a non-linear prediction formulation, unlike the conventionally used linear prediction formulation. The minimization property of the Hopfield Neural Network is exploited to solve this problem. The optimum non- linear predictor coefficients obtained from this minimization algorithm are used to estimate the EOG artefact which is then subtracted from tlic corrupted EEG signal, sample by sample, to get the artefact minimized signal. Thus the power and efficacy of the NN approach have been exploited for the purpose of enhancement of CXG signals in the presence of EOG artefacts.
\end{abstract}

\section{Introduction}

By applying electrodes to the cerebral cortex or scalp and connecting these to an amplifier, continuous oscillations of electrical potentials of various form, amplitude and frequency can he recorded. Recording of these oscillations are called electroenceplialograni (EEG), and the method itself electroencephalography. These signals reflect the activities in the underlying brain structure and particularly in the cerebral cortex below the scalp surface. Electroencephalography is used to study the relationship between the changes of electrical potentials in the different areas of cortex and subcortical formations. Four basic types of rhythms (Alpha $(\alpha)$, Beta $(\beta)$, Theta $(\theta)$ and Delta $(6))$ are distinguished according to the frequency content of the measured electrical activity. Alpha rhythm is a wave with a frequency range of 8 to $13 \mathrm{~Hz}$ and amplitudes upto 50 microvolts. Beta rhythm is characterised by wave frequencies in the range 14 to $30 \mathrm{~Hz}$ and amplitudes of 20 or 25 microvolts. Theta rhythm consists of waves with frequencies between 4 and $7 \mathrm{~Hz}$ and amplitudes of 100 or 150 microvolts. Delta rhythm is characterised by slow waves with frequencies between 0.5 and $3 \mathrm{~Hz}$ and amplitudes of 250 to 300 microvolts. In addition to these activities, EEG also contains transients like spikes, spindles etc. [1].

EEG is often severely contaminated with extracerebral signals called artefacts. They may be technical in nature due to the measurement apparatus or physiological in nature due to the subject himself. One of the main disturbances due to the subject, is ocular artefacts resulting from movements in thc ocular systems (eyeballs, eyelids etc.) [2] [3] and the corresponding electrical activity, is called the electrooculogram (EOG). This extracerebral signal, called EOG artefact severely affects the performance of the EEG processing systems, the aim of which is to extract valid information from the signals and present it in a more convenient and objective form for interpretation. The presence of ocular artefacts in EEG signal makes it difficult to automate the analysis of EEG by computer. Effective elimination of artefacts from the collected data is an essential step in preparing data for analysis. This necessitates the removal of EOG signals from contaminated EEG signals and this forms an important part in the computer processing of EEG signals.

Several methods have been reported in the literature for the removal and control of EOG artefacts from contaminated EEG signals. The simplest method is the rejection method in which the data which contain significant ocular artefacts is discarded [4]. This can lead to an unacceptable amount of loss of data and the rejection of the segments could make the data unrepresentative [5] [6]. On-line rejection has also been achieved in the frequency domain [7]. To reduce the amount of data lost by the rejection method, the subjects are often asked to fix their eyes (eye fixation method) on a target and sometimes to avoid blinking or moving their eyes 
at critical times during the experiment [8], but this procedure is cumbersome. The method suggested by McCallum and Walter [9] employs a floating common reference. This method successfully attenuates vertical eye movement artefacts in mid-line recordings. A method suggested by Girton and Kamiya [10] subtracts a percentage of the horizontai and vertical EOG's from the time domain EEG signals. Corby and Kopell [11] and Hillyard and Galambos [12] have suggested off-line methods where eye movements and eye blinks are individually isolated and removed with different fractions of the EOG's. Whitton et al. [13] have described an off-line EOG subtraction method in the frequency domain. Woestenburg et al. [14] applied regression methods in the frequency domain to account for the frequency dependent amplitude and phase characteristics of the ocular artefacts. Barlow and Remond [15] have described a more sophisticated on-line correction technique.

Quilter et al, [16] showed that the eye movement artefacts due to either random eye movements or blinks could be estimated from the measured EEGs and EOGs using a computerised crosscorrelation procedure. Verleger et al. [5] and Fortgens and De Bruin [17] computed the eye movement artefact by applying the method of least squares to EOG channels. However, this method of ocular artefact removal results in distortion of EEG responses [19][20]. Jervis et al. [21] have developed a microprocessor based ocular artefact remover. Ifeacher et al. [22] have suggested a recursive least squares (RLS) algorithm for the on-line removal of ocular artefacts. A knowledge-based enhancement of human EEG signals was also reported by Ifeacher et al [23]. Reduction of eye movement artefact from corrupted EEG signals has also been achieved by using adaptive filters with fast RLS algorithm [24].

Eventhough various methods have been reported in the literature for the minimization of EOG artefacts from corrupted EEG signals, the power and efficacy of the Neural Network (NN) approach has not been exploited so far for this purpose. In this paper, we are proposing a NN approach to the enhancement of EEG signals in the presence of EOG artefacts. We translate the EEG enhancement problem into the optimization framework by forming an appropriate cost function. The minimization property of the Hopfield Neural Network (HNN) is exploited to find the solution to the above optimization problem.

An Artificial Neural Network (ANN) consists of many highly interconnected, simple and similar processing elements, operating in parallel. Since the structure and operation of these elements arc similar to those of biological neurons, these elements are called neurons. High computational rates and robustness are the two features which result from such an architecture of the ANNs [25]: In general, ANNs can be classified into two classes; Feedforward ANNs and Feedback ANNs. One of the most important applications of the Feedback type ANNs is in solving optimization problems. For example, the ability of Hopfield ANN [26][27] to provide fast and collectively computed solutions to difficult optimization problems is well established in the literature [28] [29] [30].

\section{HNN Formulation of the Artefact Minimization Problem}

Figure 1 shows the general block representation of the. artefact minimization scheme that we have used here.

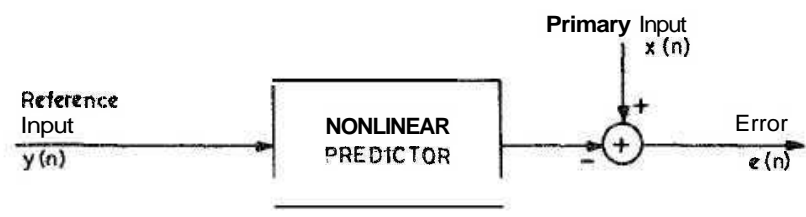

Fig $t$ Block diagram of tho artefact minimization scheme

Let $x(n)$ be the primary input and $y(n)$ be the reference input to the artefact minimizer. The predicted value of the $n^{\text {th }}$ sample of the reference input is given as the output of the nonlinear predictor model

$$
\hat{y}(n)=\mathbf{a}^{T} \mathbf{y}(n)+\mathbf{y}^{T}(n) \mathbf{A y}(n)
$$

where

$$
\begin{aligned}
\mathrm{a} & ==\left[\begin{array}{llll}
a_{1} & a_{2} & \cdots & a_{P}
\end{array}\right]^{T} \\
A & =\left[\begin{array}{lll}
a_{i, j}
\end{array}\right] \text { with } a_{i j}=0 \text { for } j \leq i, \quad,, \jmath=1,2, \ldots P
\end{aligned}
$$

and

$$
\mathbf{y}(n)=[y(n-1) y(n-2) \quad \cdots \quad y(n-P)]_{T}
$$

where a, and $a_{i j}$ are predictor coefficients, and $P$ is the order of the nonlinear predictor.

Due to the presence of the nonlinear term in the above predictor model, traditional algorithms cannot be applied to find the optimum predictor coefficients. By optimal, we mean that the energy of the unpredictable part is minimimum. The efficacy of the HNN is exploited here to obtain the optimum predictor coefficients.

The theory of HNN says that the system of neurons connected in a feedback configuration evolves in such a way that the stable stationary states of the network correspond to the local minima of it mathematical quantity called as the energy function (or Lyapunov function) of the network. So, setting up a NN to optimize a given cost function involves establishing a one-to-one correspondance between the various quantities in the given cost function and the energy function of the network. In the conventional NN 
approach, the model of the neuron is fixed a priori and the connection strengths of the neurons are assigned appropriate values by comparing the energy function and the cost function. But in our approach, the model of the neuron evolves as a direct consequence of the nature of the cost function to be minimized.

The energy function (cost function) which is central to the working principle of HNN is formulated for our problem as follows:

The error, $e(n)$ is given by

$$
e(n)=x(n)-\hat{y}(n)
$$

The cost function $E$ is defined as

$$
\boldsymbol{E}=\underset{n=1}{\boldsymbol{E}} e^{2}(n)
$$

where $N$ is the data length. The cost function $\mathrm{E}$ is minimized to get the optimum predictor coefficients. It may be noted that $\mathrm{E}$ is always positive.

Now, to obtain the structure of the HNN which solves the minimization problem, we proceed as below using the Lyapunov stability approach. We can accept $E$ as the energy function for the network to be obtained, provided the network dynamics are such . that the time derivative of $E$ is negative.

$$
d E-{ }_{i=1}^{P} d E \quad d a ;+a_{i} d t+\sum_{j=1}^{P-1} \sum_{k=j+1}^{P} \frac{d E}{\partial a_{j k}} \cdot \frac{d a_{j k}}{d t}
$$

Now, suppose we define the dynamics of the neurons as

$$
\frac{d a,}{d t}=-\frac{\partial E}{d a,}
$$

and

$$
\frac{d a_{j k}}{d t}=-\frac{\partial E}{\partial a_{j k}}
$$

giving

$$
\frac{d E}{d t}=-\sum_{i}\left[\frac{d a_{i}}{d t}\right]^{2}-\mathbf{E}_{j} \mathbf{E}_{k}\left[\frac{d a_{j k}}{d t}\right]^{2}
$$

From the above equation (2.7) we note that

$$
\begin{aligned}
& \frac{d E}{\overrightarrow{d E}}<0 \text { if } \frac{d}{\ddot{d E}} \neq 0, \frac{d a_{j k}}{d t} \neq 0 \text { for at least one } i, j \text { or } k \\
& \frac{d t}{d t}-0 \text { if } \frac{d a_{i}}{d t}=0, \frac{d a_{j k}}{d t}=0 \text { for all } i, j \text { and } k
\end{aligned}
$$

This implies that the $\mathrm{NN}$ with dynamics given by eqns $(2.5,2.6)$ has its stable stationary points at the local minima of $E$.

Using eqns(2.3),(2.5) and (2.6), the dynamics of the $\mathrm{NN}$ is now given by the following system of ordinary differential equations.

$$
\begin{gathered}
\frac{d a}{d t}=-2\left\{\mathbf{R a}+\sum_{n=1}^{N}\left[\mathbf{y}^{T}(n) \mathbf{A y}(n) \cdot \mathbf{y}(n)\right]-\mathbf{r}\right\} \\
d \mathbf{A}=\sum_{n=1}^{N}\left[\mathbf{y}^{T}(n) \mathbf{A y}(n) \cdot \mathbf{y}(n) \mathbf{y}^{T}(n)+\mathbf{a}^{T} \mathbf{y}(n) \cdot \mathbf{y}(n) \mathbf{y}^{T}(n)\right. \\
\left.\mathbf{d t}=-x(n) \cdot \mathbf{y}(n) \mathbf{y}^{T}(n)\right]
\end{gathered}
$$

where

$$
\mathbf{R}=\sum_{n=1}^{N} \mathbf{y}(n) \mathbf{y}^{T}(n)
$$

and

$$
\mathbf{r}=\sum_{n=1}^{N} x(n) \mathbf{y}(n)
$$

Using the minimization technique, the predictor coefficients are calculated for a block of data by solving the system of ordinary differential equations. Runge-Kutta method of numerical integration has been used to solve this system of equations.

\section{Results and Discussion}

The algorithm has been developed and used for the minimization of EOG artefacts from corrupted EEC signals. One derivation of the EOG signal is recorded from the left eye position on separate channel simultaneously with the EOG contaminated EEG signal from $F p_{1}$ position on the scalp using a Nihon-Khoden EEG machine. These signals are digitized at $100 \mathrm{~Hz}$ and low pass filtered at $35 \mathrm{~Hz}$ using a linear phase finite impulse response digital filter. The EOG contaminated EEG signal is used as the primary input and EOG signal as the reference input to the artefact minimizer. Five seconds of data $(N=500)$ was used. The ,predictor order $P$ was chosen to be 6 . Figure 2(a) shows the variation of the contaminated EEG with time in which the large amplitude low frequency activity is due to the eye movements. Figure 2(b) is the corrected EEG signal, obtained using the $\mathrm{T}$ algorithm, in which the EOG artefact has been effectively minimized.

In order to make an assessment of the effectiveness of the proposed NN algorithm in removing the EOG artefact from contaminated EEG signals, we have calculated the smoothed power spectrum of these signals using linear prediction (LP) technique [31]. Figure 3(a) shows the LP spectrum of the contaminated EEG signal which shows a peaky response at about $2.5 \mathrm{~Hz}$ implying the presence of low frequency EOG artefact,. In Figure 3(b) (which is the LP spectrum of the corrected EEG signal) the peaky response at low frequency is effectively reduced implying that the proposed $\mathrm{NN}$ algorithm is very effective in minimizing the EOG artefact from contaminated EEG signals.

\section{Conclusions}

We have translated the EEG enhancement problem into the optimization framework by choosing the sum of the squared prediction errors as the cost function. The minimization property of the HNN is exploited to find the solution to this optimization problem. The optimum nonlinear predictor coefficients obtained from the minimization algorithm are used to estimate the artefact that contaminates the primary input of the artefact minimizer. 
This estimate of the artefact is successively subtracted from the primary input signal, sample by sample, to get the artefact minimized signal. The time plots as also the LP spectrum show that the proposed method effectively minimizes the EOG artefacts from contaminated EEG signals. Thus the power and efficacy of NN approach has been exploited for the purpose of enhancement of EEG signals in the presence of EOG artefact.

\section{References}

[1 ] Storm Van Leeuwen, W., Bickford, R., Brazier, M., Cobb, W.A., Dondey, H., Gastaut, H., Gloor, P., Henry, C.E., Hess, R., Knott, J.R., Kugler, J., Lairy, G.C., Loeb, C., Magnus, O., Daurella, L.O., Petsche, N., Schwab, R., Walter, W.G., and Widen, L., "Proposal for an EEG terminology by terminology committee of the International federation for Electroencephalography and Clinical Neurophysiology", Electro. Clin. Neurophysiol., Vol.20, 1966, pp.306-310.

[2 ] Geddes. L.A and Baker, L.E, Principles of applied biomedical instrumentation. Wiley and Sons. New York, 1975, pp.509-517.

[3 ] Hillyard, S.A, "Methodological issues in CNV research", In Bioelectric Recording Techniques Part B. Electro. Human Brain Potentials, Thomson, R.F and Patterson, M.M (Eds.), Academic Press, 1974, pp.259-280.

[4 | Barlow, J.S. "Computerized clinical electroencephalography in perspective", IEEE Trans. BME. Vol.26, No:7, 1979, pp.377-391.

[5 ] Verleger, R., Gasser, T. and Mocks, J., "Correction of EOG artefacts in event related potentials of the EEG: aspects of reliability and validity", Psychophysiology, Vol.19, 1982,pp.472480.

[6 ) Gratton, G., Coles, M.G.H. and Donchin, E., "A new method of off- line removal of ocular artefacts", Electro. Clin. Neurophysiol., Vol.55, 1983, pp. 468-484.

[7 ] Gevins, A.S., Yeager, C.L., Zeitlin, G.M., Ancoli, S and Dedon, M.F., "011-line computer rejection of EEG artefact. Electro. Clin. Keurophysiol., Vol.42, 1977, pp. 267-274.

[8 ] Papakostopoulos, D., Winter, A. and Newton, P., "New techniques for the control of eye potential artefacts in multichannel CNV recordings", Electro. Clin. Neurophysiol., Vol.34, 1973, pp 651-653.

[9 ] McCallum. W.C and Walter, W.G. "The effects of attention and distraction on the contingent negetive variation in normal and neurotic subjects", Electro. Clin. Neurophysiol., Vol.25, 1968, pp. 319-329.

[10 ] Girton, D.G and Kamiya, J., "A simple on-line technique for removing eye movement artefacts from the EEG." Electro. Clin. Keurophysiol., Vol.34, 1973,pp. 212-216.

[11 ] Corby, J.C and Kopell, B.S. "Differential contributions of blinlts and vertical eye movements as artefacts in EEC recording", Psychophysiology. Vol.9, 1972, pp. 640-644.

[12 ] Hillyard, D.G and Galambose, R. "Eye movement artefact in the CNV", Electro. Clin. Neurophysiol., Vol.28, 1970, pp. 173-182.

[13 ] Whitton, J.L., Leu, F. and Moldofsky, H ., "A spectral method for removing eye movement artefacts from the EEG". Electro. Clin. Neurophysiol., Vol.44, 1978, pp. 735-741

[14 ] Woestenburg, J.C., Verbaten, M.N. and Slagen, J.L., "Removal of eye movement artefact from the EEG by regression analysis in the frequency domain", Biol. Psychol., Vol. 16, 1983, pp. 127-147.

[15 ] Barlow, J.S and Remond, A. "Eye movement artefact nulling in EEG's by multichannel on-line EOG subtraction", Electro. Clin. Neurophysiol., Vol.55, 1981,pp. 418-423

[16 ] Quilter, P.M., MacGillivray, B.B. and Wadbrook, D.G., "The removal of eye movement artefact from EEG signals using correlation technique, In random signal analysis", , IEE Conf. Publ.. Vol.159. 1977, pp. 93-100.

[17 ] Fortgens, C and De Bruin, M.P., "Removal of eye movement and ECG artefacts from non-cephalic reference EEG", Electro. Clin. Neurophysiol., Vol.56, 1983, pp. 90-96.

[18 ] Jervis, B.W., Nichols, M.J., Allen, E.M., Hudson, N.R. and Johnson, T.E., "Tlhe assessment of two methods for removing eye movement artefact from EEG", Electro. Clin. Neurophysiol., Vol.61, 1985, pp. 444-452.

[19 ] Jervis, B.W., Coelho, M. and Morgan, C.W., "Effect on EEG responses of removing ocular artefacts by proportional EOG subtraction", Med. and Biol. Engg. and Computing, Vol.27, 1989, pp. 484-490.

[20 ] Jervis, B.W., Ifeacher, E.C., and Allen, E.M.. "The removal of ocular artefacts from the Electroencephalogram: a review", Med. and Biol. Engg and Computing, Vol.26, 1988, pp. 2-12.

[21 ] Jervis, B.W., Ifeacher, E.C., Allen, E.M., Morris, E.L. and Hudson, N.R., "Removal of ocular artefact from the human 
EEG", IEEE 7th Annual Conf. of the Engg. in Med. and Biol. Soceity, Chicago, USA, 1985, pp.101-107.

[22 ] Ifeacher, E.C., Jervis, B.W., Morris, E.L., Allen, E.M. and Hudson, N.R., "New on-line method for removing ocular artefacts from EEG signals", $\quad$ Med. and Biol. Engg. and Computing, Vol.24, 1986, pp. 356-364.

[23 ] Ifeacher, E.C., Hellyar, M.T., Mapps, D.J. and Allen, E.M., "Knowledge based enhancement of human EEG signals", IEE Proc.. Vo1.137. Pt.F. 1990,pp. 302-310.

[24 ] Sadasivan, P.K and Narayana Dutt, D., "Eye movement artefact rejection in EEG signals by adaptive cancellation technique", Proc. National Conf. on Elect. Circuits and Systems, Roorkee, 1989, pp.' 254-256.

[25 ] Lippman, R.P., "An introduction to computing with Neural Nets", IEEE Acoust. Speech. Signal Processing Magazine, V01.4, 1987, pp.4-22.

[26 ] Hopfield, J.J., "Neural Networks and physical systems with emergent collective computational abilities", Proc. Natl. Acad. Sci., USA, Vol.79, 1982,pp 2554-2558.

[27 ] Hopfield, J.J., "Neurons with graded response have collective computational properties like those of two-state neurons", Proc. Natl. Acad. Sci., USA, Vol.81, 1984,pp 3088-3092.

[28 ] Hopfield, J.J., and Tank, D.W., "Neural computation of decision in optimization problems", Biological Cybernetics, Vol.52, 1985, pp. 141- 152.

[29 ] Hopfield, J.J., and Tank, D.W., "Computing with Neural circuits; A model", Science. Vol.233, 1986, pp. 625-633,

[30 ] Tank, D.W., and Hopfield, J.J., "Simple Neural optimization networks : An A/D converter, signal decision circuit and linear programming circuit", IEEE Trans. Circuits and Systems, Vol.CAS-33, 1986,pp 533-541.

[31 ] Makhoul, J., "Linear prediction: A tutorial review", Proc.IEEE. Vol.3, 1975, pp. 561-580.
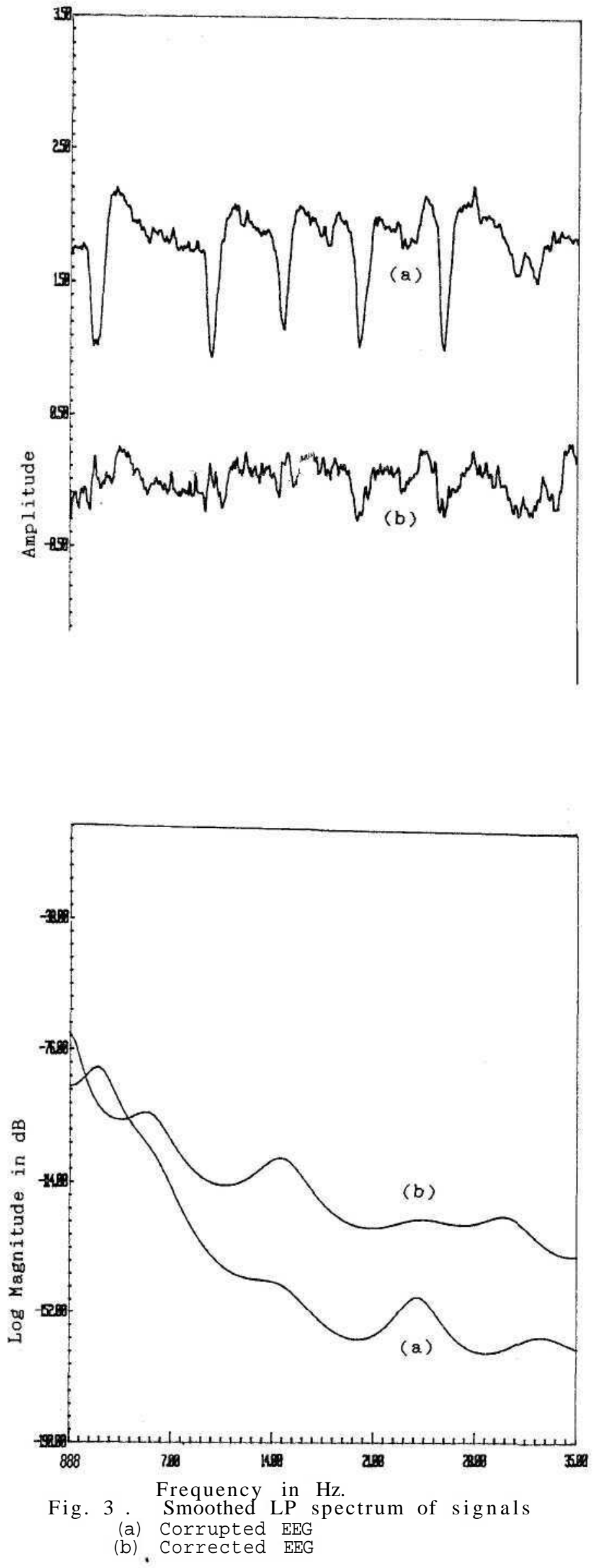